\title{
Characterization of Salmonella enterica Isolates from Diseased Poultry in Northern China between 2014 and 2018
}

\author{
Jun Wang ${ }^{1}{ }^{\mathbb{D}}$, Jinxin $\mathrm{Li}^{1}{ }^{1}$, Fengli Liu ${ }^{1}$, Yongyou Cheng ${ }^{2}$ and Jingliang $\mathrm{Su}^{1, *}$ \\ 1 Key Laboratory of Animal Epidemiology of the Ministry of Agriculture, College of Veterinary Medicine, \\ China Agricultural University, Beijing 100193, China; luodian825@cau.edu.cn (J.W.); \\ b20193050453@cau.edu.cn (J.L.); bs20193050480@cau.edu.cn (F.L.) \\ 2 College of Food and Pharmaceutical Engineering, Guiyang University, Guiyang City 550002, \\ Guizhou Province, China; chengang01@caas.cn \\ * Correspondence: suzhang@cau.edu.cn; Tel.: +86-10-6273-2312
}

Received: 16 January 2020; Accepted: 29 January 2020; Published: 4 February 2020

\begin{abstract}
Salmonella infection not only causes acute and chronic diseases in poultry flocks, but the infected poultry are among the most important reservoirs for a variety of Salmonella serovars frequently transmitted to humans. This study aimed to investigate the occurrence of Salmonella spp. in local poultry farms in China. Samples $(\mathrm{n}=4255)$, including dead-in-shell embryos, culled day-old-hatchings and 1- to 4-week-old diseased birds, were collected for Salmonella culture from broiler chicken, meat-type duck and pigeon farms in northern China between 2014 and 2018. A total of 103 Salmonella were isolated. S. enterica serovar Enteritidis and S. Typhimurium were the most prevalent serovars, representing $53.4 \%$ and $34.9 \%$ of the isolates, respectively. Serovar diversity was the highest in ducks, with the $S$. Apeyeme being isolated for the first time from duck tissues. All isolates were characterized by multilocus sequence typing (MLST) and pulsed-field gel electrophoresis (PFGE). MLST showed that all S. Enteritidis isolates shared the same sequence type (ST11), and Typhimurium showed several rare STs in addition to ST19. In comparison, PFGE showed better discrimination for S. Enteritidis and $S$. Typhimurium isolates, with nine distinct pulsotypes being observed. The isolates exhibited varying degrees of resistance to 15 tested antimicrobials and identified $S$. Enteritidis isolates $(98.18 \%)$ with multiple antimicrobial resistance were a cause for concern. Our data on invasive Salmonella infection in meat-type poultry in local farms can be used to identify sources and factors associated with Salmonella spread in poultry and the associated food chain.
\end{abstract}

Keywords: Salmonella; duck; chicken; pigeon; MLST; PFGE; antimicrobial resistance

\section{Introduction}

Salmonella spp. are one of the leading bacterial pathogens in human beings and domesticated and wild animals [1,2]. It has been estimated that Salmonella enterocolitis resulted in 95.1 million cases and 50,771 deaths in 2017 [3-5]. In addition to diarrhea, 535,000 cases of nontyphoidal Salmonella invasive disease occurred, as well as an estimated 77,500 deaths [6]. Animals are the primary reservoirs of Salmonella; especially poultry and poultry products are among the most frequently implicated animal sources for Salmonella entering the human food supply. In China, 22.16\% of bacterial foodborne illnesses were caused by Salmonella between 1994 and 2005 [7]. Contaminated poultry foods including meat and eggs are an important route of transmission of Salmonella from animals to humans [8,9].

China is a large producer of poultry, especially with the largest production of meat-type ducks and pigeons $[10,11]$. In contrast to intensively produced broiler chickens, commercial ducks and pigeons are more frequently reared in semi-intensive housing systems using simple accommodations with 
access to outside pens and bathing water or sands. In practice, Salmonella can be introduced to poultry flocks from multiple sources, e.g., from the environment, feed and vectors due to the lack of efficient biosecurity measures [12]. Poultry can become infected with different Salmonella serotypes with high morbidity and mortality during the first three weeks of their life, and may also become carriers which can disseminate bacteria spread horizontally within flocks, or vertically to progeny via contaminating breeding eggs, leading to embryo mortality or rapid death of newly hatched birds. The prevalence of Salmonella infection in Chinese poultry farms has been widely described $[13,14]$. In the chicken sector, voluntary control in breeding flocks in combination with national statutory elimination of $S$. Pullorum has reduced Salmonella infection. However, ducks and pigeons are not included and limited information on Salmonella prevalence is available. Concerning poultry production and food safety, it is important to understand the prevalence of the pathogen in terms of its control.

The aim of the present work was to study the epizootic status of Salmonella in the local meat-type poultry farms. Strains isolated from dead-in-shell embryos, culled day-old birds and tissue samples of diseased poultry between 2014 and 2018 were characterized by: (1) serotyping, (2) genotyping using multilocus sequence typing (MLST) and pulsed-field gel electrophoresis (PFGE) and (3) antimicrobial susceptibility testing.

\section{Results}

\subsection{Isolation and Serotyping of Salmonella}

Based on the growth characteristics on MacConkey and XLD (Xylose Lysine-Deoxycholate) agar plates, presumptive Salmonella colonies were subcultured and screened by PCR based on the Salmonella-specific invA gene [15]. From 4255 clinical poultry samples, a total of 103 (2.42\%) Salmonella were isolated (Table 1). Using the classical serotyping agglutination method, these isolates were assigned to eight serotypes distributed in seven $O$ groups. Among these isolates, the highest occurring serotype was $S$. Enteritidis $(\mathrm{n}=55)$, followed by $S$. Typhimurium $(\mathrm{n}=36), S$. Anatum $(\mathrm{n}=3), S$. Apeyeme $(\mathrm{n}=3), S$. Kottbus $(\mathrm{n}=2)$, S. Senftenberg $(\mathrm{n}=2), S$. Montevideo $(\mathrm{n}=1)$ and $S$. Pullorum $(\mathrm{n}=1)$. The high frequency of the two serotypes Enteritidis and Typhimurium in the clinical samples is in agreement with their overall prevalence in poultry flocks in the European Union [16]. The distribution of the serotypes varied with host species. For pigeon samples, only serotype Typhimurium was isolated, whereas almost all isolates recovered from chicken were $S$. Enteritidis except for one $S$. Pullorum isolate. The greatest serotype diversity was observed among isolates from duck tissue samples. Of note, $S$. Apeyeme was recovered from duck origin samples for the first time in China.

Table 1. Salmonella identification of diseased birds.

\begin{tabular}{|c|c|c|c|c|c|c|c|}
\hline \multirow[t]{2}{*}{ Serovar } & \multicolumn{3}{|c|}{ Duck } & \multicolumn{2}{|c|}{ Chicken } & \multirow{2}{*}{$\begin{array}{c}\text { Pigeon } \\
\text { Liver or Brain } \\
\text { of Dead } \\
\text { Pigeon } \\
(\mathrm{n}=55)\end{array}$} & \multirow{2}{*}{$\begin{array}{l}\text { Total } \\
4255\end{array}$} \\
\hline & $\begin{array}{c}\text { Dead-in-Shell } \\
\text { Embryo } \\
(\mathrm{n}=1028)\end{array}$ & $\begin{array}{l}\text { Day-old } \\
\text { Hatching } \\
(\mathrm{n}=1400)\end{array}$ & $\begin{array}{c}\text { One- to } \\
\text { Four-Week-Old } \\
\text { Duck } \\
(\mathrm{n}=\mathbf{1 3 0 7 )}\end{array}$ & $\begin{array}{c}\text { Dead-in-Shell } \\
\text { Embryo } \\
(n=450)\end{array}$ & $\begin{array}{l}\text { One- to } \\
\text { Four-Week-Old } \\
\text { Chicken } \\
(n=15)\end{array}$ & & \\
\hline Enteritidis & 1 & - & 33 & 19 & 2 & - & 55 \\
\hline Typhimurium & 2 & - & 22 & - & - & 12 & 36 \\
\hline Apeyeme & - & 3 & - & - & - & - & 3 \\
\hline Anatum & 2 & 1 & - & - & - & - & 3 \\
\hline Senftenberg & - & - & 2 & - & - & - & 2 \\
\hline Kottbus & - & - & 2 & - & - & - & 2 \\
\hline Montevideo & - & 1 & - & - & - & - & 1 \\
\hline Pullorum & - & - & - & - & 1 & - & 1 \\
\hline
\end{tabular}

\subsection{Multilocus Sequence Analysis}

MLST typing based on the comparison of internal sequences of seven housekeeping gene fragments was carried out on the 103 isolates. With exception of $S$. Typhimurium, sequence type assignment exhibited a one-to-one relationship with serovar (Figure 1). All 55 S. Enteritidis isolates were assigned to ST11. By contrast, the 36 S. Typhimurium isolates were subtyped into five STs, namely ST19 (23/36), 
ST1922 (7/36), ST1544 (4/36), ST128 (1/36) and ST2211 (1/36). It was noted that the STs represented in our collection were all single-locus variants of ST19, with only one nucleotide difference (Table 2). Interestingly, seven isolates consisting of ST1922 were exclusively isolated from diseased pigeons.

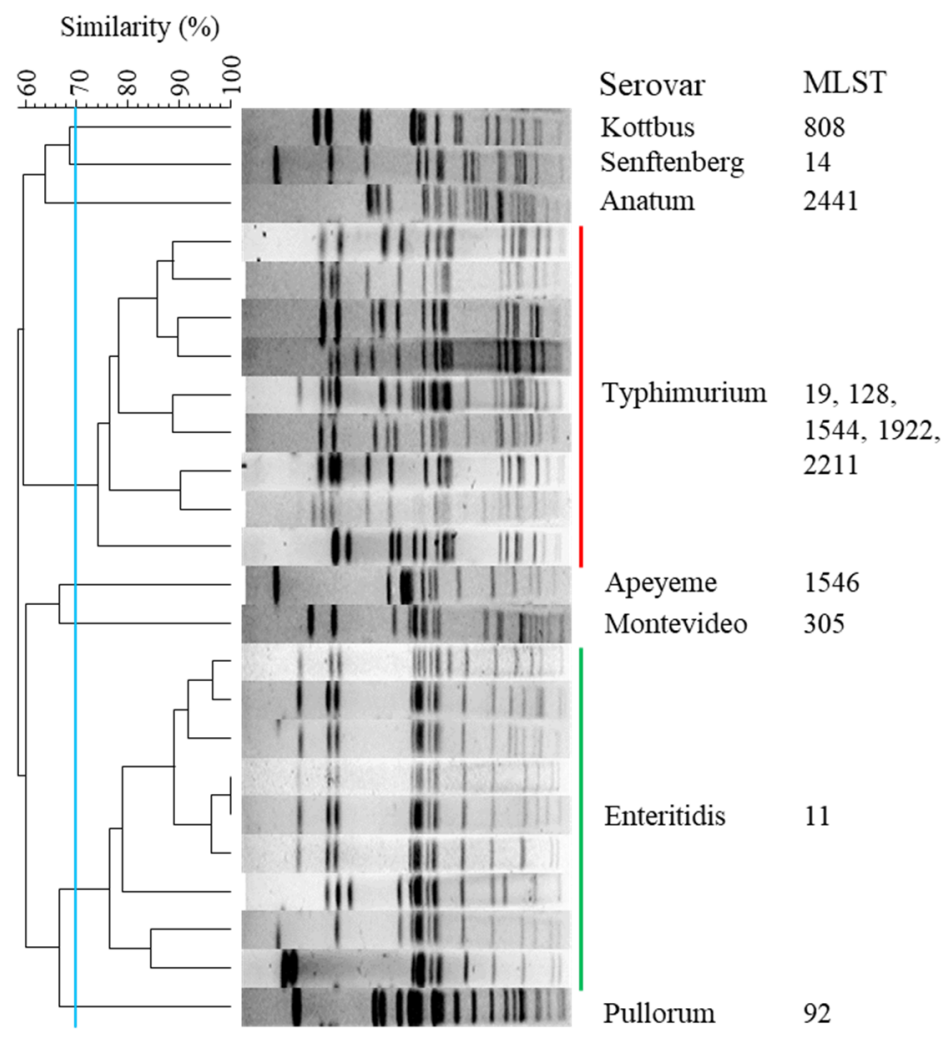

Figure 1. XbaI-pulsed-field gel electrophoresis (PFGE) profile and multilocus sequence typing (MLST) of eight Salmonella serotypes. A total of 103 isolates are assigned into eight groups with lower than $70 \%$ similarity. The isolates of Typhimurium and Enteritidis are grouped into nine profiles with at least one band difference. With the exception of serovar Typhimurium, the remaining serotypes display the same MLST of within-serotypes.

Table 2. Nucleotide discrimination of S. Typhimurium MLST.

\begin{tabular}{ccccc}
\hline ST of $S$. Typhimurium & \multicolumn{2}{c}{ hisD } & purE & sucA \\
\hline 19 & $482 \mathrm{G}$ & $539 \mathrm{G}$ & $81 \mathrm{~T}$ & $478 \mathrm{G}$ \\
128 & - & - & - & $478 \mathrm{C}$ \\
1544 & - & $539 \mathrm{~A}$ & - & - \\
1922 & $482 \mathrm{~A}$ & - & - & - \\
2211 & - & - & $81 \mathrm{C}$ & - \\
\hline
\end{tabular}

\subsection{PFGE Analysis}

When the genomic DNA of all Salmonella isolates was digested with the XbaI enzyme and separated by PFGE, the DNA fragments had a good separation, giving 11 to 19 bands per isolate. The 103 Salmonella isolates were successfully assigned into 8 groups representing different serotypes. The genetic similarity among these groups was lower than $70 \%$ (Figure 1).

With respect to $S$. Enteritidis, 55 isolates exhibited genetic relatedness ranging from $79.1 \%$ to $100 \%$. Defining subtypes as those with at least $85 \%$-similarity banding patterns resulted in 3 subtypes with at least a three bands difference. All $55 \mathrm{~S}$. Enteritidis could be grouped within three main PFGE subtypes at an $85 \%$ genetic similarity (Figure 2). For further visual analyses based on the dendrogram, isolates were regarded as having a distinct PFGE profile if they differed by at least one band from other 
isolates. By these criteria, these isolates were primarily grouped into nine pulsotypes (labeled PSe1 through PSe9) (Figure 2) with within-profile similarity $\geq 99.5 \%$. PSe 1, 2, 3, 4 and 9 were consisted by duck isolates. The profiles PSe 5, 6, 7 and 8 were observed in isolates from chicken and duck samples, containing a majority of our $S$. enteritidis isolates (duck $=23$, chicken $=19$ ). It was evident that PFGE analysis yielded a greater differentiation than that of the MLST on the S. Enteritidis subtyping in this study.
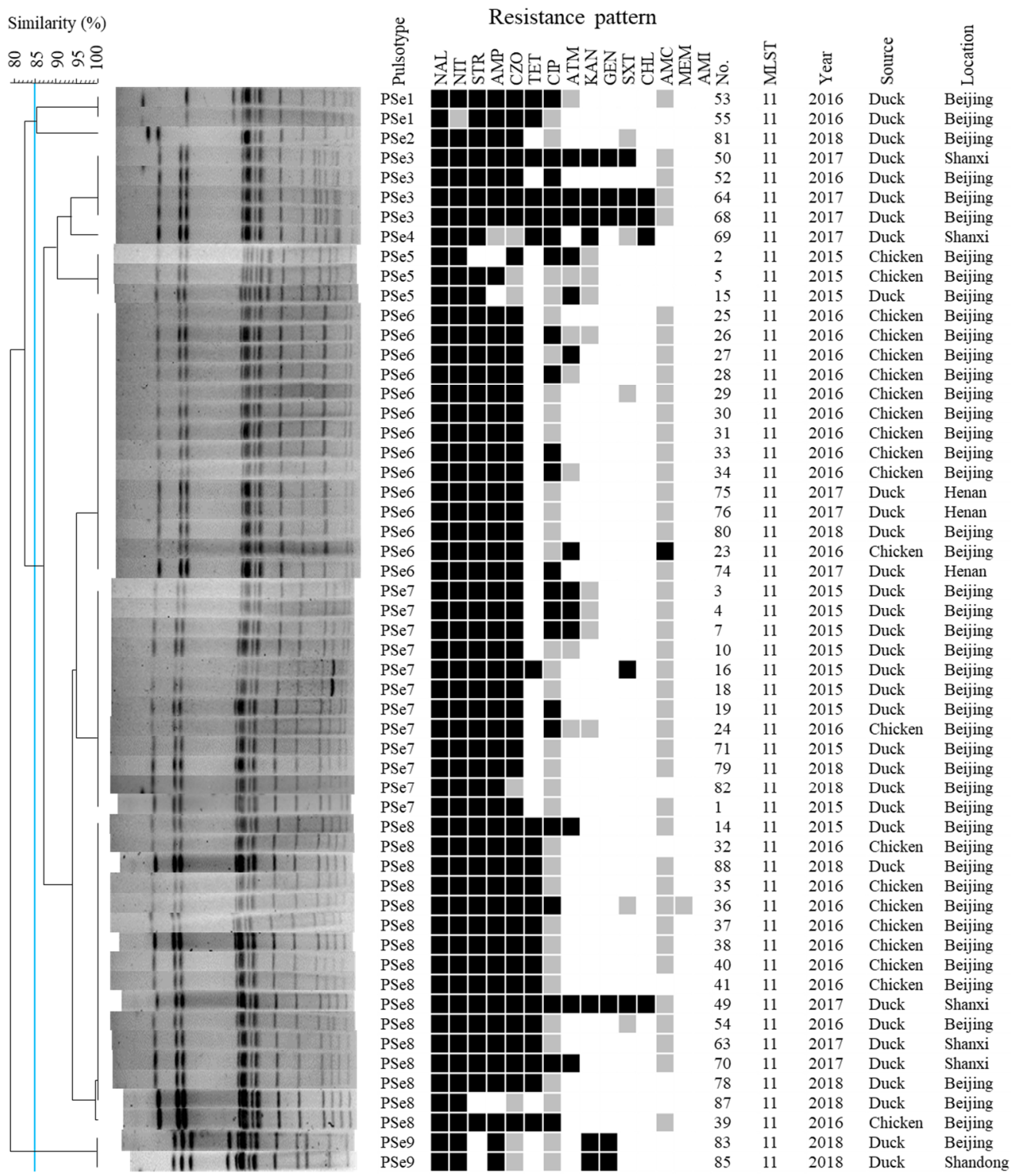

Figure 2. XbaI-PFGE profiles, antimicrobial resistance pattern and MLST of all $S$. Enteritidis isolates. For PFGE, 55 isolates were divided into three subtypes according to an $85 \%$ similarity. A black box indicates resistance against specified antimicrobial, a gray box indicates intermediate resistance and a white box indicates susceptibility. Antimicrobial designations are detailed in Section 4.

Using the same criteria, the $36 \mathrm{~S}$. Typhimurium isolates were grouped into five subtypes ( $>3$ bands difference) after XbaI digestion, and further divided into nine PFGE profiles (PSt1 through PSt9) with $\geq 1$ band difference) (Figure 3). We noted that isolates from ducks and pigeons exhibited distinct banding profiles except for one isolate from pigeon that was assigned to PSt6 with two duck-origin isolates. Most duck isolates (19/20) that were assigned to ST19 by MLST typing were grouped in PSt1 
except one, which was grouped into PSt9. PSt6 contained two duck- and one pigeon-origin ST1544 isolates, and one duck-origin ST1544 isolate displayed a distinct banding profile (PSt7). The only Typhimurium isolated from a duck sample in Henan was assigned as ST2211, exhibiting a different banding pattern (PSt3). Most pigeon isolates distributed in PSt5 (6/12) and PSt4 (3/12) with only one band difference between them. In general, XbaI PFGE generated a higher index of discrimination for both $S$. Enteritidis and $S$. Typhimurium.

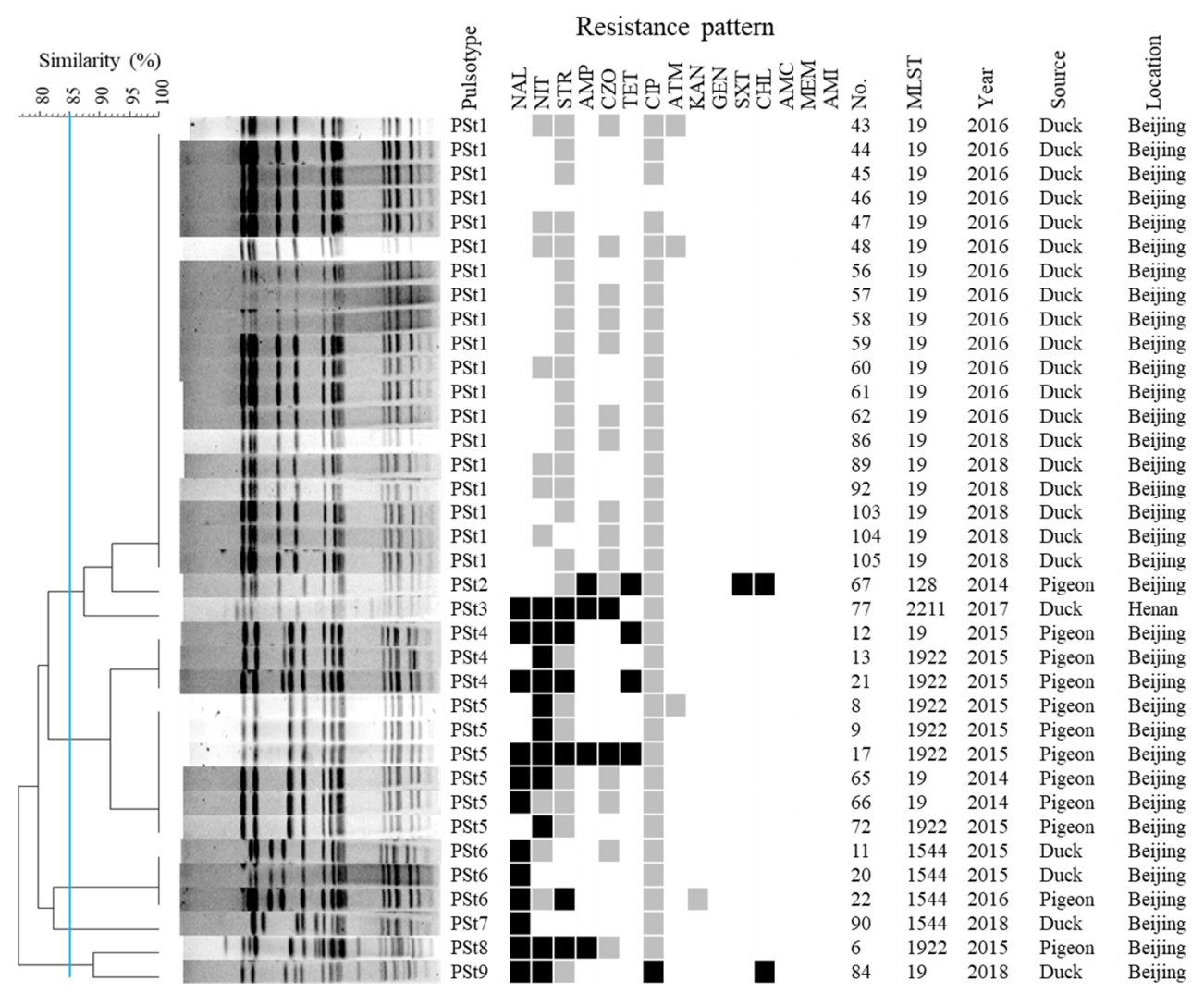

Figure 3. XbaI-PFGE profiles, antimicrobial resistance pattern and MLST of all $S$. Typhimurium isolates. For PFGE, 36 isolates were divided into three subtypes according to an $85 \%$ similarity. A black box denotes resistance against specified antimicrobial, a gray box denotes intermediate resistance and a white box denotes susceptibility. Antimicrobial designations are detailed in Section 4.

\subsection{Salmonella Isolates Displayed High Levels of Antimicrobial Resistance}

The 103 tissue-culture Salmonella isolates were tested for susceptibility to 15 antimicrobial drugs. Resistance to at least one drug was observed among 77 isolates (74.8\%), representing ten different resistance patterns (Table 3). Although no resistance was observed for the other 26 isolates, 25 of them exhibited intermediate resistance to 1 to 4 drugs, including meropenem (MEM). One isolate exhibited pan-susceptibility to all tested antimicrobials.

When stratified by serotype, $S$. Enteritidis displayed a significantly higher frequency of antimicrobial resistance than that of other serotypes. Except isolate 87, all $S$. Enteritidis isolates ( $n=54)$ were resistant to $\geq 3$ antimicrobial classes, which is considered multidrug resistance (MDR) [17]. The most frequent resistance phenotypes were nalidixic acid (NAL) (100\%), nitrofurantoin (NIT) (98.2\%), streptomycin (STR) $(92.7 \%)$, ampicillin (AMP) $(92.7 \%)$ and cefazolin (CZO) $(87.3 \%)$, followed by tetracycline (TET) (40\%), aztreonam (ATM) (23.6\%), ciprofloxacin (CIP) $(22.33 \%)$, kanamycin (KAN) $(12.7 \%)$, gentamicin (GEN) (10.9\%), trimethoprim/sulfamethoxazole (SXT) $(9.1 \%)$, chloramphenicol $(\mathrm{CHL})(7.3 \%)$ and amoxicillin/clavulanic acid (AMC) $(1.8 \%)$. The most common pattern of resistance involved NAL-NIT-STR-AMP-CZO and NAL-NIT-STR-AMP-CZO-TET, with 14 and 12 isolates, 
respectively. Association relationship between species and resistance patterns was not significantly observed. However, four isolates recovered from ducks in 2017 displayed resistance to 11 to 12 tested antimicrobials.

Table 3. Antimicrobial resistance pattern of 103 Salmonella isolates.

\begin{tabular}{|c|c|}
\hline Antimicrobial Resistance Patterns & Isolates no. \\
\hline Susceptible to all tested antimicrobials & 26 \\
\hline NAL & 7 \\
\hline NIT & 4 \\
\hline NAL-STR & 1 \\
\hline NAL-TET & 1 \\
\hline NAL-NIT & 2 \\
\hline NAL-STR-KAN & 1 \\
\hline NAL-NIT-CHL & 1 \\
\hline NAL-NIT-STR-ATM & 1 \\
\hline NAL-NIT-STR-AMP & 2 \\
\hline NAL-NIT-STR-ATM & 1 \\
\hline AMP-TET-SXT-CHL & 1 \\
\hline NAL-NIT-TET-STR & 2 \\
\hline NAL-NIT-CIP-CHL & 1 \\
\hline NAL-NIT-CZO-CIP-ATM & 1 \\
\hline NAL-STR-AMP-CZO-TET & 1 \\
\hline NAL-NIT- STR-AMP-CZO & 14 \\
\hline NAL-NIT-AMP-KAN-GEN & 2 \\
\hline NAL-NIT-STR-AMP-CZO-CIP & 8 \\
\hline NAL-NIT-STR-AMP-CZO-TET & 12 \\
\hline NAL-NIT-STR-AMP-CZO-CIP-ATM & 3 \\
\hline NAL-NIT-STR-AMP-CZO-TET-SXT & 1 \\
\hline NAL-NIT-STR-AMP-CZO-ATM-AMC & 1 \\
\hline NAL-NIT-STR-AMP-TET-CIP-FAZ & 3 \\
\hline NAL-NIT-STR-TET-CIP-KAN-CHL & 1 \\
\hline NAL-NIT-STR-AMP-CZO-TET-CIP-ATM & 2 \\
\hline NAL-NIT-STR-AMP-CZO-TET-CIP-ATM-KAN-GEN-SXT & 1 \\
\hline NAL-NIT-STR-AMP-CZO-TET-CIP-ATM-KAN-GEN-SXT-CHL & 3 \\
\hline
\end{tabular}

NAL: nalidixic acid; NIT: nitrofurantoin; STR: streptomycin; AMP: ampicillin; CZO: cefazolin; TET: tetracycline; CIP: ciprofloxacin; ATM: aztreonam; KAN: kanamycin; GEN: gentamicin; SXT: trimethoprim/sulfamethoxazole; CHL: chloramphenicol; AMC: amoxicillin/clavulanic acid; MEM: meropenem; AMI: meropenem.

Compared with the collection of $S$. Enteritidis, $S$. Typhimurium isolates exhibited a significantly lower frequency of antimicrobial resistance. 17 of the 36 isolates were resistant to at least one antimicrobial. Antimicrobial resistance was predominantly observed in pigeon-origin isolates. All pigeon isolates (12/12) were resistant to at least one of the tested antimicrobials and five of them exhibited multidrug resistance ( $\geq 3$ antimicrobial classes). By contrast, five duck isolates (5/24) had antimicrobial resistance and two were resistant to three (NAL-NIT-CHL) and five (NAL-NIT-STR-AMP-CZO) antimicrobials. 


\section{Discussion}

In this study, we analyze 103 Salmonella isolates from poultry tissue with clinical disease, which provides relevant results to understand the epidemiology of Salmonella infection in meat-type poultry farms. Invasive Salmonella isolates involving the death of hatching embryos, hatched-duck culling, fatal infection of table ducklings and broiler chickens in parts of northern China, between 2014 and 2018, were typified at the serovar level and by an approach involving several molecular typing methods. The serovars identified in this study indicate the diversity of Salmonella in commercial poultry flocks in northern China. S. Enteritidis and $S$. Typhimurium were the most frequently isolated, consistent with previous reports from China and some European countries $[18,19]$. Of the remaining isolates, $S$. Anatum, $S$. Senftenberg, $S$. Kottbus and $S$. Montevideo were also commonly isolated from poultry-based products and humans in China [20-22]. This result reflects the common occurrence of these serovars in meat-type poultry, highlighting the reservoirs being maintained in the poultry practices. It is notable that $S$. Apeyeme was isolated from clinical poultry samples for the first time. Although the pathogenicity of $S$. Apeyeme needs further investigation, its emergence as a zoonotic pathogen is of concern because it has been isolated from human, shellfish and apple snail (https://enterobase.warwick.ac.uk/species/index/senterica).

In the present study, 74 (71.84\%) Salmonella strains were isolated from tissues of diseased poultry, highlighting the association with clinical infection on these poultry farms. Remarkably, the majority of these isolates distributed in diseased duck $(59,57.28 \%)$ and pigeon tissues $(12,11.65 \%)$. The relatively high frequency of invasive infection of Salmonella in commercial meat-type duck and pigeon flocks may largely relate to semi-open rearing systems that lack effective biosecurity. Birds are reared on the ground or on slatted plastic flooring, whereby Salmonella can be introduced into flocks from many sources and transmitted efficiently within a flock. Isolation of seven serovars from diseased duck samples also indicated a high level of Salmonella diversity in commercial duck flocks as in a previous study [23]. Duck might serve as a reservoir of various Salmonella serotypes. It has been reported that infection of ducklings most likely happens at the hatchery [24]; however, the isolation rate of Salmonella spp. from dead-in-shell embryos and culled 1-day-old ducklings in our study was $0.41 \%(10 / 2428)$, which was significantly lower than those reported in previous studies $(2.1 \%$ and $12.2 \%)[13,23]$. This difference might be influenced by the environmental conditions on the sampled farms, where breeding eggs were fully washed and sanitized with chlorine bleach before entering the hatchery. By contrast, 1-day-old chicks were found to have a Salmonella prevalence of $4.2 \%$ (19/450). This high prevalence might be in relation to sample collections. These samples were targeted for Salmonella because broiler chickens derived from these hatcheries were experiencing Salmonella infection during the test period.

From the diseased meat-type pigeon samples, only $S$. Typhimurium was identified in this study, and it remains to be seen whether these isolates belong to a host-adapted serovar as reported $[25,26]$. However, care should be taken when interpreting serotype data from broiler chickens and pigeons because all pigeon Typhimurium isolates originated from dead pigeons submitted for clinical diagnosis, and may not, therefore, represent the true distribution of the serotypes in these populations. Previous reports have revealed that five serovars (Typhimurium, Enteritidis, Derby, Brandenburg and Pomona) were recovered from meat, liver, intestine and cloacal swab of pigeons in China, Madrid and Flanders [27-29]. As for the distribution of Salmonella serotypes in three chicken farms, only $S$. Enteritidis and S. Pullorum assigned into the same $\mathrm{O}$ group were isolated. Along with the result, slide agglutination tests of 143 sera collected from these farms were performed using inactivated S. Pullorum antigen. $40(27.97 \%)$ sera showed positive reaction. This result was consistent with Salmonella isolation.

MLST and PFGE have been widely used to subtype Salmonella isolates for genetic relatedness and epidemiological evaluation. In this study, both MLST and PFGE subtyping grouped isolates together into the representing serovar. All 55 Enteritidis isolates were assigned into ST11, a vastly distributed clone across the world $[14,30,31]$. However, PFGE analysis showed that the 55 isolates displayed nine banding patterns distinguishable by at least one band difference (Figure 2); this banding pattern 
similarity was higher than that of $S$. Enteritidis isolates from breeder chickens reported recently [32]. No obviously epidemiological relatedness was explored from the same and different samples. Although the vast majority of Enteritidis isolates were grouped in subtype E2, the distinct patterns of isolates in subtypes E1 and E3 indicated that PFGE provided greater discrimination for subtyping $S$. Enteritidis than did MLST [33].

In this study, the majority of $S$. Typhimurium isolates were grouped into ST19 (63.89\%), the most common sequence types isolated from humans and animal-based food products across the world [34,35]. Moreover, ST128, ST1544 and ST2211 have been identified from human and animal samples in China and the United Kingdom [28,36]. Perhaps circulatory transmission generated between contaminated poultry meat and human beings. Interestingly, the ST1922 in our collection consisted of seven isolates originating exclusively from diseased pigeons. A previous study has revealed that ST1922 Typhimurium was originally isolated from liver of pigeon sold in one market in Sanya, China [37]. To our knowledge, ST1922 of Typhimurium has been specifically isolated from pigeon samples until now. Further analysis may be needed to explore the restrictive relationship between the ST type and pigeon host. Besides, other ST types of Typhimurium were also recovered from meat of pigeon recently [28]. Using PFGE subtyping the 36 Typhimurium isolates were classified into five subtypes with a $<85 \%$ similarity (Figure 3). Most of the duck- and pigeon-origin Typhimurium isolates were distributed in subtype 1 and subtype 2, respectively, suggesting some association between PFGE and host-origin. On the other hand, 19 S. Typhimurium isolates shared identical PFGE patterns in PSt1, suggesting the circulation of clonality among the $S$. Typhimurium in the local duck farms.

The emergence and development of antimicrobial resistance of Salmonella isolates from animals and their products may be due to a variety of reasons [38]. The qualitative results determined by the disk diffusion method suggested that Salmonella isolates in our collection exhibited varying resistance to the 15 tested antimicrobials. It should be noted that antibiotics are frequently used on poultry farms, which may be the cause of the resistance to the tested antibiotics. In this work, high rates of resistance to nalidixic acid $(65.05 \%)$, nitrofurantoin $(63.11 \%)$, streptomycin $(55.34 \%)$, ampicillin $(53.40 \%)$ and cefazolin $(48.54 \%)$ were observed. This result agreed with previous reports in China, Iran and the United States [28,39,40]. Beyond that, high resistance of Enteritidis has been reported frequently $[39,41]$, but $100 \%$ (55/55) resistance was somewhat unexpected, especially the extremely high frequency (54/55) of MDR. These agents containing nalidixic acid, nitrofurantoin, streptomycin, ampicillin and cefazolin have been extensively used in poultry practices in China, which was noted by the Chinese veterinary pharmacopoeia and the report [42]. High prevalence of antimicrobial resistance should draw our attention. Further studies are needed to assess factors that might play a role in the development of AMR (antimicrobial resistance) in commercial poultry practices in northern China. However, our study might have overestimated the frequency of AMR because some samples came from birds that might already have been treated with antimicrobials. S. Typhimurium in general exhibited decreased resistance to most of the 15 antimicrobials. This result was inconsistent with other reports in China [43,44]. Nevertheless, more resistant isolates were found in diseased pigeon samples than in duck-origin ones. We speculate that the frequency of utilization and types of agents were quite different in these farms through clinical interrogation, which is supported by the study. [19].

In conclusion, our study showed the presence of Salmonella serovars in clinically diseased birds in local poultry flocks. High frequency isolation of the most common serovars Enteritidis and Typhimurium indicates that meat-type poultry could be reservoirs for zoonotic Salmonella strains. The extremely high rate of antimicrobial resistance in clinical Salmonella Enteritidis isolates is of great concern for antimicrobial use in poultry practice, and may pose a threat to human health. The observed association between the serotypes and molecular subtypes of Salmonella isolates in this study suggest that combining results obtained from all three methods might provide more useful epidemiological information related to infection. 


\section{Materials and Methods}

\subsection{Salmonella Isolation and Serotyping}

Isolation and identification of Salmonella were conducted following the OIE (World Organisation for Animal Health) manual for salmonellosis diagnostic test with partial modifications. One- to four-week-old ducks, broiler chickens and pigeons from poultry farms distributed in Henan, Shanxi, Shandong, Hebei and Beijing from 2014 to 2018 were sampled for diagnosis. For birds with observable gross lesions (e.g., perihepatitis and swollen liver at autopsy), the surface of liver was branded with scalding surgical blade and the skull was disinfected with $75 \%$ ethanol and opened using sterile scissor. Disinfected tissue was then streaked onto MacConkey agar plates and tryptic soy agar plates (Becton, Dickinson and Co, Sparks, MD, USA) by inoculation loop. After incubation at $37^{\circ} \mathrm{C}$ for 18 to $24 \mathrm{~h}$, suspected colonies from every plate were picked and subcultured on MacConkey plates. For dead-in-shell embryos and 1-day-old hatchings culled at the hatchery, the whole liver and cecum of every one were aseptically obtained and sliced, pooled (in groups of 5) and placed into $100 \mathrm{~mL}$ of buffered peptone water (BPW) (Beijing Landbridge Technology Co., Ltd., Beijing, China). Following incubation at $37^{\circ} \mathrm{C}$ for $24 \mathrm{~h}, 1 \mathrm{~mL}$ of culture was transferred into $10 \mathrm{~mL}$ of Rappaport-Vassiliadis enrichment broth and incubated at $37^{\circ} \mathrm{C}$ for $24 \mathrm{~h}$. Then one loop of the broth culture was streaked onto XyloseLysine-Deoxycholate Agar (XLD) plate (Becton, Dickinson and Co, Sparks, MD, USA) and characteristic colonies were picked and subcultured after incubation at $37^{\circ} \mathrm{C}$ for $24 \mathrm{~h}$. To screen for Salmonella, the suspected colonies were screened by PCR assay with the primer pair (5'-GTGAAATTATCGCCACGTTCGGGCAA-3'/5'-TCATCGCACCGTCAAAGGAACC-3') based on the Salmonella invA gene. Selected Salmonella were subcultured and serotyped by slide agglutination using commercial $\mathrm{O}$ and $\mathrm{H}$ antisera to distinguish Salmonella serovars (Tianrun Bio-Pharmaceutical, Ningbo, China) in accordance with the White-Kauffmann-Le Minor scheme [45]. All identified isolates were stored at $-80^{\circ} \mathrm{C}$ for further use.

\subsection{Multilocus Sequence Typing}

MLST for the seven housekeeping genes, including thrA, purE, sucA, hisD, aroC, hemD and dnaN was performed according to protocols described previously [46]. Briefly, all strains stored at $-80^{\circ} \mathrm{C}$ were streaked onto tryptic soy agar and incubated at $37^{\circ} \mathrm{C}$ for $12 \mathrm{~h}$; then one colony was picked and inoculated into tryptic soy broth. After overnight incubation, $50 \mu \mathrm{l}$ of bacterial culture was collected and boiled at $95{ }^{\circ} \mathrm{C}$ for $10 \mathrm{~min}$. The suspension was briefly centrifuged to remove cellular debris and the supernatant was used as a DNA template for the PCR assay. All PCR amplification was performed in a volume of $50 \mu \mathrm{l}$ containing $25 \mu \mathrm{l}$ of $2 \times$ Taq PCR Mix (GenStar, Beijing, China), $4 \mu \mathrm{l}$ of template, $2 \mu \mathrm{l}$ of each $25 \mu \mathrm{M}$ primer and $17 \mu \mathrm{l}$ of sterile $\mathrm{ddH}_{2} \mathrm{O}$. The PCR reactions were conducted with the following thermal cycling profile: initial denaturation at $94{ }^{\circ} \mathrm{C}$ for $5 \mathrm{~min}$ followed by 30 cycles of $94{ }^{\circ} \mathrm{C}$ for $30 \mathrm{~s}, 56{ }^{\circ} \mathrm{C}$ for $30 \mathrm{~s}, 72{ }^{\circ} \mathrm{C}$ for $30 \mathrm{~s}$ and an additional extension at $72{ }^{\circ} \mathrm{C}$ for $7 \mathrm{~min}$. The PCR products were gel-purified and subjected to bidirectional DNA sequencing using an Applied Biosystems ABI3730 sequencer (Meiji Biological Medicine Technology Co., Ltd. Shanghai, China). Sequences were aligned using Megalign (Lasergene 10, DNAStar, Madison, WI, USA). The nucleotide sequence for each gene fragment was submitted to the Salmonella MLST database (http://mlst.warwick.ac.uk/mlst/dbs/Senterica) to obtain the specific Salmonella sequence type.

\subsection{Pulsed-field Gel Electrophoresis}

PFGE of 103 isolates was performed following the PulseNet protocol with modification [47]. Briefly, $200 \mu \mathrm{l}$ of the overnight culture of each isolate was added to agarose for plug preparation. Plugs were lysed at $54^{\circ} \mathrm{C}$ for $2 \mathrm{~h}$, and the obtained DNA was digested with $50 \mathrm{U}$ of $\mathrm{XbaI}$ (TaKaRa, Dalian, China) at $37^{\circ} \mathrm{C}$ for $4 \mathrm{~h}$. The restriction fragments were separated in a $0.5 \times$ Tris-borate-EDTA buffer at $14{ }^{\circ} \mathrm{C}$ for $18 \mathrm{~h}$ using a Chef Mapper electrophoresis system (Bio-Rad Laboratories, Hercules, CA, USA) with switch times of $2.16 \mathrm{~s}$ and $63.8 \mathrm{~s}$. The gels were stained with GoldenView and visualized 
under UV transillumination. Salmonella Braenderup H9812 was used as the standard molecular marker. Images were analyzed using the InfoQuest software version 4.5 (Bio-Rad Laboratories). The PFGE patterns were analyzed according to the Dice similarity coefficient method using the BioNumerics software (version 7.6; Applied Maths, Kortrijk, Belgium). The unweighted pair group method with arithmetic mean (UPGMA) was selected to construct a dendrogram with a position tolerance of $1.5 \%$ and optimization of $1.5 \%$.

\subsection{Antimicrobial Resistance Test}

The susceptibility of 103 isolates to a panel of 15 antimicrobials was determined using the Kirby-Bauer disk diffusion method according to the Clinical and Laboratory Standards Institute (CLSI) recommendation [48]. The antimicrobials and corresponding concentration included ampicillin (AMP, $10 \mu \mathrm{g}$ ), amoxicillin/clavulanic acid (AMC, 20/10 $\mu \mathrm{g}$ ), cefazolin (CZO, $30 \mu \mathrm{g})$, meropenem (MEM, $10 \mu \mathrm{g})$, aztreonam (ATM, $30 \mu \mathrm{g})$, kanamycin (KAN, $30 \mu \mathrm{g})$, gentamicin (GEN, $10 \mu \mathrm{g})$, streptomycin (STR, $10 \mu \mathrm{g})$, amikacin (AMI, $30 \mu \mathrm{g}$ ), tetracycline (TET, $30 \mu \mathrm{g}$ ), trimethoprim/sulfamethoxazole (SXT, 1.25/23.75 $\mu \mathrm{g}$ ), ciprofloxacin (CIP, $5 \mu \mathrm{g}$ ), nalidixic acid (NAL, $30 \mu \mathrm{g}$ ), chloramphenicol (CHL, $30 \mu \mathrm{g}$ ) and nitrofurantoin (NIT, $300 \mu \mathrm{g}$ ). Escherichia coli ATCC25922 was used as the quality control.

Author Contributions: Conceptualization, J.W. and J.S.; methodology, J.W., J.L. and F.L.; writing-original draft preparation, J.W. and J.S.; writing-review and editing, J.W., Y.C. and J.S. All authors have read and agreed to the published version of the manuscript.

Funding: This research was partially funded by the Beijing Municipal Science and Technology Commission, grant number No. Z181100009318007.

Acknowledgments: The authors would like to thank Yang Wang at the College of Veterinary Medicine, China Agricultural University, for the help in the PFGE experiment. We thank Louise Adam, ELS(D), from Liwen Bianji, Edanz Editing China (www.liwenbianji.cn/ac) for editing the English text of a draft of this manuscript.

Conflicts of Interest: The authors declare no conflict of interest. The funders had no role in the design of the study; in the collection, analyses, or interpretation of data; in the writing of the manuscript, or in the decision to publish the results.

Ethics Statement: All animal experiments in this study were carried out in accordance with the China Agricultural University Animal Ethic Committee (03022020-2), which complied with the guidelines of the Review of Welfare and Ethics of Laboratory Animals approved by the Beijing Municipality Administration Office of Laboratory Animals.

\section{References}

1. Majowicz, S.E.; Musto, J.; Scallan, E.; Angulo, F.J.; Kirk, M.; O’Brien, S.J.; Jones, T.F.; Fazil, A.; Hoekstra, R.M. The global burden of nontyphoidal Salmonella gastroenteritis. Clin. Infect. Dis. 2010, 50, 882-889. [CrossRef]

2. Simpson, K.M.J.; Hill-Cawthorne, G.A.; Ward, M.P.; Mor, S.M. Diversity of Salmonella serotypes from humans, food, domestic animals and wildlife in New South Wales, Australia. BMC Infect. Dis. 2018, 18, 623. [CrossRef]

3. GBD 2017 Causes of Death Collaborators. Global, regional, and national age-sex-specific mortality for 282 causes of death in 195 countries and territories, 1980-2017: a systematic analysis for the Global Burden of Disease Study 2017. Lancet 2018, 392, 1736-1788. [CrossRef]

4. GBD 2017 DALYs and HALE Collaborators. Global, regional, and national disability-adjusted life-years (DALYs) for 359 diseases and injuries and healthy life expectancy (HALE) for 195 countries and territories, 1990-2017: a systematic analysis for the Global Burden of Disease Study 2017. Lancet 2018, 392, 1859-1922. [CrossRef]

5. GBD 2017 Disease and Injury Incidence and Prevalence Collaborators. Global, regional, and national incidence, prevalence, and years lived with disability for 354 diseases and injuries for 195 countries and territories, 1990-2017: a systematic analysis for the Global Burden of Disease Study 2017. Lancet 2018, 392, 1789-1858. [CrossRef]

6. GBD 2017 Non-Typhoidal Salmonella Invasive Disease Collaborators. The global burden of non-typhoidal Salmonella invasive disease: a systematic analysis for the Global Burden of Disease Study 2017. The Lancet Infect. Dis. 2019, 19, 1312-1324. [CrossRef] 
7. Wang, S.; Duan, H.; Zhang, W.; Li, J.W. Analysis of bacterial foodborne disease outbreaks in China between 1994 and 2005. FEMS Immunol. Med. Microbiol. 2007, 51, 8-13. [CrossRef] [PubMed]

8. Antunes, P.; Mourao, J.; Campos, J.; Peixe, L. Salmonellosis: the role of poultry meat. Clin. Microbiol. Infect. 2016, 22, 110-121. [CrossRef]

9. Chousalkar, K.; Gole, V.C. Salmonellosis acquired from poultry. Curr. Opin. Infect. Dis. 2016, 29, 514-519. [CrossRef]

10. Gilbert, M.; Nicolas, G.; Cinardi, G.; Van Boeckel, T.P.; Vanwambeke, S.O.; Wint, G.R.W.; Robinson, T.P. Global distribution data for cattle, buffaloes, horses, sheep, goats, pigs, chickens and ducks in 2010. Sci. Data 2018, 5, 180227. [CrossRef]

11. Liang, Y.Y.; Liang, Y.; Chen, Y.Z.; Wang, Z.W. Current situation and development prospect of China's pigeon industry. South China Rural Area 2018, 34, 30-32. (In Chinese) [CrossRef]

12. Soria, M.C.; Soria, M.A.; Bueno, D.J.; Godano, E.I.; Gomez, S.C.; ViaButron, I.A.; Padin, V.M.; Roge, A.D. Salmonella spp. contamination in commercial layer hen farms using different types of samples and detection methods. Poult. Sci. 2017, 96, 2820-2830. [CrossRef] [PubMed]

13. Li, R.; Lai, J.; Wang, Y.; Liu, S.; Li, Y.; Liu, K.; Shen, J.; Wu, C. Prevalence and characterization of Salmonella species isolated from pigs, ducks and chickens in Sichuan Province, China. Int. J. Food Microbiol. 2013, 163, 14-18. [CrossRef] [PubMed]

14. Yang, J.; Gao, S.; Chang, Y.; Su, M.; Xie, Y.; Sun, S. Occurrence and Characterization of Salmonella Isolated from Large-Scale Breeder Farms in Shandong Province, China. Biomed. Res. Int. 2019, 2019, 8159567. [CrossRef]

15. Rahn, K.; De Grandis, S.A.; Clarke, R.C.; McEwen, S.A.; Galán, J.E.; Ginocchio, C.; Curtiss, R.; Gyles, C.L. Amplification of an invA gene sequence of Salmonella typhimurium by polymerase chain reaction as a specific method of detection of Salmonella. Mol. Cell. Probes 1992, 6, 271-279. [CrossRef]

16. EFSA and ECDC (European Food Safety Authority and European Centre for Disease Prevention and Control). The European Union summary report on trends and sources of zoonoses, zoonotic agents and food-borne outbreaks in 2017. EFSA J. 2018, 16, e05500. [CrossRef]

17. Magiorakos, A.P.; Srinivasan, A.; Carey, R.B.; Carmeli, Y.; Falagas, M.E.; Giske, C.G.; Harbarth, S.; Hindler, J.F.; Kahlmeter, G.; Olsson-Liljequist, B.; et al. Multidrug-resistant, extensively drug-resistant and pandrug-resistant bacteria: an international expert proposal for interim standard definitions for acquired resistance. Clin. Microbiol. Infect. 2012, 18, 268-281. [CrossRef]

18. Osimani, A.; Aquilanti, L.; Clementi, F. Salmonellosis associated with mass catering: a survey of European Union cases over a 15-year period. Epidemiol. Infect. 2016, 144, 3000-3012. [CrossRef]

19. Zeng, Y.B.; Xiong, L.G.; Tan, M.F.; Li, H.Q.; Yan, H.; Zhang, L.; Yin, D.F.; Kang, Z.F.; Wei, Q.P.; Luo, L.G. Prevalence and Antimicrobial Resistance of Salmonella in Pork, Chicken, and Duck from Retail Markets of China. Foodborne Pathog. Dis. 2019, 16, 339-345. [CrossRef]

20. Wollin, R. A study of invasiveness of different Salmonella serovars based on analysis of the Enter-net database. Euro. Surveill. 2007, 12, 3275. [CrossRef]

21. Cai, Y.; Tao, J.; Jiao, Y.; Fei, X.; Zhou, L.; Wang, Y.; Zheng, H.; Pan, Z.; Jiao, X. Phenotypic characteristics and genotypic correlation between Salmonella isolates from a slaughterhouse and retail markets in Yangzhou, China. Int. J. Food Microbiol. 2016, 222, 56-64. [CrossRef] [PubMed]

22. Yang, X.; Wu, Q.; Zhang, J.; Huang, J.; Chen, L.; Wu, S.; Zeng, H.; Wang, J.; Chen, M.; Wu, H.; et al. Prevalence, Bacterial Load, and Antimicrobial Resistance of Salmonella Serovars Isolated From Retail Meat and Meat Products in China. Front. Microbiol. 2019, 10, 2121. [CrossRef] [PubMed]

23. Yang, J.; Ju, Z.; Yang, Y.; Zhao, X.; Jiang, Z.; Sun, S. Serotype, antimicrobial susceptibility and genotype profiles of Salmonella isolated from duck farms and a slaughterhouse in Shandong province, China. BMC Microbiol. 2019, 19, 202. [CrossRef] [PubMed]

24. Martelli, F.; Birch, C.; Davies, R.H. Observations on the distribution and control of Salmonella in commercial duck hatcheries in the UK. Avian Pathol. 2016, 45, 261-266. [CrossRef]

25. Rabsch, W.; Andrews, H.L.; Kingsley, R.A.; Prager, R.; Tschape, H.; Adams, L.G.; Baumler, A.J. Salmonella enterica serotype Typhimurium and its host-adapted variants. Infect. Immun. 2002, 70, 2249-2255. [CrossRef] 
26. Hoelzer, K.; Soyer, Y.; Rodriguez-Rivera, L.D.; Cummings, K.J.; McDonough, P.L.; Schoonmaker-Bopp, D.J.; Root, T.P.; Dumas, N.B.; Warnick, L.D.; Grohn, Y.T.; et al. The prevalence of multidrug resistance is higher among bovine than human Salmonella enterica serotype Newport, Typhimurium, and 4,5,12:i:- isolates in the United States but differs by serotype and geographic region. Appl. Environ. Microbiol. 2010, 76, 5947-5959. [CrossRef]

27. Haesendonck, R.; Rasschaert, G.; Martel, A.; Verbrugghe, E.; Heyndrickx, M.; Haesebrouck, F.; Pasmans, F. Feral pigeons: A reservoir of zoonotic Salmonella Enteritidis strains? Vet. Microbiol. 2016, 195, 101-103. [CrossRef]

28. Yang, X.; Huang, J.; Zhang, Y.; Liu, S.; Chen, L.; Xiao, C.; Zeng, H.; Wei, X.; Gu, Q.; Li, Y.; et al. Prevalence, abundance, serovars and antimicrobial resistance of Salmonella isolated from retail raw poultry meat in China. Sci. Total Environ. 2020, 713, 136385. [CrossRef]

29. Perez-Sancho, M.; García-Seco, T.; Porrero, C.; García, N.; Gomez-Barrero, S.; Cámara, J.M.; Domínguez, L.; Álvarez, J. A ten-year-surveillance program of zoonotic pathogens in feral pigeons in the City of Madrid (2005-2014): The importance of a systematic pest control. Res. Vet. Sci. 2020, 128, 293-298. [CrossRef]

30. Noda, T.; Murakami, K.; Asai, T.; Etoh, Y.; Ishihara, T.; Kuroki, T.; Horikawa, K.; Fujimoto, S. Multi-locus sequence typing of Salmonella enterica subsp. enterica serovar Enteritidis strains in Japan between 1973 and 2004. Acta Vet. Scand. 2011, 53, 38. [CrossRef]

31. Retamal, P.; Fresno, M.; Dougnac, C.; Gutierrez, S.; Gornall, V.; Vidal, R.; Vernal, R.; Pujol, M.; Barreto, M.; Gonzalez-Acuna, D.; et al. Genetic and phenotypic evidence of the Salmonella enterica serotype Enteritidis human-animal interface in Chile. Front. Microbiol. 2015, 6, 464. [CrossRef] [PubMed]

32. Fei, X.; Yin, K.; Yin, C.; Hu, Y.; Li, J.; Zhou, Z.; Tian, Y.; Geng, S.; Chen, X.; Pan, Z.; et al. Analyses of prevalence and molecular typing reveal the spread of antimicrobial-resistant Salmonella infection across two breeder chicken farms. Poult. Sci. 2018, 97, 4374-4383. [CrossRef] [PubMed]

33. Papadopoulos, T.; Petridou, E.; Zdragas, A.; Mandilara, G.; Nair, S.; Peters, T.; Chattaway, M.; de Pinna, E.; Passiotou, M.; Vatopoulos, A. Comparative study of all Salmonella enterica serovar Enteritidis strains isolated from food and food animals in Greece from 2008 to 2010 with clinical isolates. Eur. J. Clin. Microbiol. Infect. Dis. 2016, 35, 741-746. [CrossRef] [PubMed]

34. Murgia, M.; Bouchrif, B.; Timinouni, M.; Al-Qahtani, A.; Al-Ahdal, M.N.; Cappuccinelli, P.; Rubino, S.; Paglietti, B. Antibiotic resistance determinants and genetic analysis of Salmonella enterica isolated from food in Morocco. Int. J. Food Microbiol. 2015, 215, 31-39. [CrossRef] [PubMed]

35. Ktari, S.; Ksibi, B.; Gharsallah, H.; Mnif, B.; Maalej, S.; Rhimi, F.; Hammami, A. Molecular epidemiological characteristics of Salmonella enterica serovars Enteritidis, Typhimurium and Livingstone strains isolated in a Tunisian university hospital. APMIS 2016, 124, 194-200. [CrossRef]

36. Sun, J.; Ke, B.; Huang, Y.; He, D.; Li, X.; Liang, Z.; Ke, C. The Molecular Epidemiological Characteristics and Genetic Diversity of Salmonella Typhimurium in Guangdong, China, 2007-2011. PloS ONE 2014, 9, e113145. [CrossRef]

37. Yang, X.; Wu, Q.; Zhang, J.; Huang, J.; Guo, W.; Cai, S. Prevalence and Characterization of Monophasic Salmonella Serovar 1,4,[5],12:i:- of Food Origin in China. PLoS ONE 2015, 10, e0137967. [CrossRef] [PubMed]

38. Florez-Cuadrado, D.; Moreno, M.A.; Ugarte-Ruiz, M.; Dominguez, L. Antimicrobial Resistance in the Food Chain in the European Union. Adv. Food Nutr. Res. 2018, 86, 115-136. [CrossRef]

39. Fardsanei, F.; Soltan Dallal, M.M.; Douraghi, M.; Memariani, H.; Bakhshi, B.; Zahraei Salehi, T.; Nikkhahi, F. Antimicrobial resistance, virulence genes and genetic relatedness of Salmonella enterica serotype Enteritidis isolates recovered from human gastroenteritis in Tehran, Iran. J. Glob. Antimicrob. Resist. 2018, 12, $220-226$. [CrossRef]

40. Velasquez, C.G.; Macklin, K.S.; Kumar, S.; Bailey, M.; Ebner, P.E.; Oliver, H.F.; Martin-Gonzalez, F.S.; Singh, M. Prevalence and antimicrobial resistance patterns of Salmonella isolated from poultry farms in southeastern United States. Poult. Sci. 2018, 97, 2144-2152. [CrossRef]

41. Wang, Y.; Yang, B.; Wu, Y.; Zhang, Z.; Meng, X.; Xi, M.; Wang, X.; Xia, X.; Shi, X.; Wang, D.; et al. Molecular characterization of Salmonella enterica serovar Enteritidis on retail raw poultry in six provinces and two National cities in China. Food Microbiol. 2015, 46, 74-80. [CrossRef] [PubMed]

42. Guo, X.; Wang, H.; Cheng, Y.; Zhang, W.; Luo, Q.; Wen, G.; Wang, G.; Shao, H.; Zhang, T. Quinolone resistance phenotype and genetic characterization of Salmonella enterica serovar Pullorum isolates in China, during 2011 to 2016. BMC Microbiol. 2018, 18, 225. [CrossRef] [PubMed] 
43. Wang, J.; Li, Y.; Xu, X.; Liang, B.; Wu, F.; Yang, X.; Ma, Q.; Yang, C.; Hu, X.; Liu, H.; et al. Antimicrobial Resistance of Salmonella enterica Serovar Typhimurium in Shanghai, China. Front. Microbiol. 2017, 8, 510. [CrossRef] [PubMed]

44. Wang, Y.; Cao, C.; Alali, W.Q.; Cui, S.; Li, F.; Zhu, J.; Wang, X.; Meng, J.; Yang, B. Distribution and Antimicrobial Susceptibility of Foodborne Salmonella Serovars in Eight Provinces in China from 2007 to 2012 (Except 2009). Foodborne Pathog. Dis. 2017, 14, 393-399. [CrossRef]

45. Gremont, P.A.D.; Weill, F.X. Antigenic Formulae of the Salmonella Serovars, 9th Edn. Paris: WHO Collaborating Center for Reference and Research on Salmonella. Institut Pasteur. 2007, 1, 1-166.

46. Kidgell, C.; Reichard, U.; Wain, J.; Linz, B.; Torpdahl, M.; Dougan, G.; Achtman, M. Salmonella typhi, the causative agent of typhoid fever, is approximately 50,000 years old. Infect. Genet. Evol. 2002, 2, 39-45. [CrossRef]

47. Ribot, E.M.; Fair, M.A.; Gautom, R.; Cameron, D.N.; Hunter, S.B.; Swaminathan, B.; Barrett, T.J. Standardization of pulsed-field gel electrophoresis protocols for the subtyping of Escherichia coli O157:H7, Salmonella, and Shigella for PulseNet. Foodborne Pathog. Dis. 2006, 3, 59-67. [CrossRef]

48. Clinical and Laboratory Standards Institute. Performance Standards for Antimicrobial Susceptibility Testing. Twenty-fifth Informational Supplement; M100-s25; Clinical and Laboratory Standards Institute: Wayne, PA, USA, 2015.

(C) 2020 by the authors. Licensee MDPI, Basel, Switzerland. This article is an open access article distributed under the terms and conditions of the Creative Commons Attribution (CC BY) license (http://creativecommons.org/licenses/by/4.0/). 